\title{
Photons as Probes of Gluon Saturation in Dilute+Dense Collisions ${ }^{\dagger}$
}

\author{
Sanjin Benić ${ }^{1}$, Kenji Fukushima ${ }^{2}$, Oscar Garcia-Montero ${ }^{3, *}$ and Raju Venugopalan ${ }^{4}$ \\ 1 Yukawa Institute for Theoretical Physics, Kyoto University, Kyoto 606-8502, Japan; sanjinb@phy.hr \\ 2 Department of Physics, The University of Tokyo, 7-3-1 Hongo, Bunkyo-ku, Tokyo 113-0033, Japan; \\ fuku@nt.phys.s.u-tokyo.ac.jp \\ 3 Institut für Theoretische Physik, Universität Heidelberg, Philosophenweg 16, 69120 Heidelberg, Germany \\ 4 Physics Department, Brookhaven National Laboratory, Bldg. 510A, Upton, NY 11973, USA; \\ raju@quark.phy.bnl.gov \\ * Correspondence: garcia@thphys.uni-heidelberg.de \\ + Presented at Hot Quarks 2018-Workshop for Young Scientists on the Physics of Ultrarelativistic \\ Nucleus-Nucleus Collisions, Texel, The Netherlands, 7-14 September 2018.
}

Published: 18 April 2019

\begin{abstract}
We use the Color Glass Condensate (CGC) effective field theory (EFT) to calculate inclusive photon production to leading order $q g \rightarrow q \gamma$, (LO), and next-to leading order $g g \rightarrow q \bar{q} \gamma$ (NLO) at LHC energies. These processes dominate the photon production at small- $x$, where $x \lesssim 0.01$ in the target and projectile protons. We show that the NLO contribution dominates at values of $x$ typical at the LHC, since its cross-section is sensitive to the gluon distributions in both protons. We perform a comparison of our results to the available inclusive photon data, from ATLAS and CMS at center-of-mass energies of 2.76 and $7 \mathrm{TeV}$. This data lies in the range $k_{\perp}>20 \mathrm{GeV}$. We show that for this range, the $k_{\perp}$-factorized cross-section converges to the full CGC EFT result, and can be used for the comparison. We find that it gives good agreement with experimental results. Our results are to be considered as a first step towards constraining unintegrated gluon distributions, which will be continued for larger systems, where coherent scatterings are enhanced.
\end{abstract}

Keywords: prompt; inclusive; photon; saturation; color glass condensate

\section{Introduction}

Photons produced in $p p$ collisions are excellent probes of the internal structures of the hadronic waveforms. At very high energies, where the gluon distributions rise, these states are described by the Color Glass Condensate [1] effective field theory. Using this framework one can compute diverse observables in the dilute-dense limit, in which one of the hadrons is sensitive to small- $x$ resummation, while the other remains in the large- $x$ regime. In this limit the parametrical leading order (LO) is given by the $q g \rightarrow q \gamma$ process [2,3], which is dominant when a hard photon is emitted from a large- $x$ valence quark before or after this scatters coherently off the target. The next-to-leading order (NLO) channel $g g \rightarrow q \bar{q} \gamma$ is dominant for high energies, in mid-rapidities, as the gluon distributions are enhanced at small- $x$. In this process, the projectile's hard gluon scatters off the target and splits into a $q \bar{q}$ pair, which then emits a photon, or it can first split into the pair, which scatters of the target gluonic shockwave.

These LO and NLO cross-sections were computed before and are given explicitly in [2-6]. In this short note we will focus on the numerical results of such a computation, and the properties that the inclusive prompt photon cross section exhibits at the LHC energies. We start by noting that one can separate the photons produced into two categories. Depending on their nature, one can have direct and fragmentation photons. One can in principle separate these two components in the LO and NLO 
contributions. However, experimentally, the only way of separating them is to impose an isolation cut as in ref. [7]. For this, we convolute the inclusive cross-section with an isolation cone given by $\theta\left(\sqrt{\left(\eta_{\gamma}-\eta\right)^{2}+\left(\phi_{\gamma}-\phi\right)^{2}}-R\right)$, where $\theta$ is the Heaviside step function, and $\eta$ and $\phi$ are the rapidity and azimuthal angle of the (anti) quark and $\eta_{\gamma}$ and $\phi_{\gamma}$ are the respective photon rapidity and transverse angle ${ }^{1}$. For the experimental data $R=0.4$ has been used, while estimating that $10 \%$ of the remaining cross-section is given by fragmentation photons [8].

\section{Results}

We used the CTEQ6M distribution set [9] for the valence quarks computation in LO contribution. Dipole evolution was given by the running coupling Balitsky-Kovchegov (rcBK) equation, which is a good approximation to the general expression given by the Balitsky-JIMWLK hierarchy. The dipole initial condition at $x=0.01$ was set by the McLerran-Venugopalan (MV) model, with an anomalous dimension $\gamma=1$, an initial saturation scale at $x_{0}$ of $Q_{0}^{2}=0.2 \mathrm{GeV}^{2}$ and the infrared cutoff of $\Lambda_{I R}=0.241 \mathrm{GeV}$. For more details, see ref. [10]. The rcBK evolution is used to find the dipoles for $x<x_{0}$. For large- $x$ values, we used the matching procedure given in ref. [11] where the unintegrated gluon distributions (UGD) are matched to the gluon partonic distribution function (PDF). This procedure also sets the proton effective radius to be $R_{p}=0.48 \mathrm{fm}$, and thus the transverse area is set to $S_{\perp}=7.24 \mathrm{mb}$. We set the quark masses to be $m_{u}=m_{d}=0.005 \mathrm{GeV}, m_{s}=0.095 \mathrm{GeV}$, $m_{c}=1.3 \mathrm{GeV}$ and $m_{b}=4.5 \mathrm{GeV}$. The quark mass parameters still have some freedom, and we will discuss the error induced by this further on. The cross sections were computed using the VEGAS Monte Carlo integration routine (more details can be found in ref. [6]). A comparison between the full CGC and the $k_{\perp}$-factorized result is shown in Figure 1, where at low $k_{\perp}$ factorization is clearly broken. One can see that $k_{\perp}$-factorization breaking is enhanced for decreasing quark masses and more forward rapidities. On the other hand, the effect is suppressed at high $k_{\gamma \perp}$, becoming negligible for $k_{\gamma \perp} \gtrsim 20 \mathrm{GeV}$. Since the available data for CMS and ATLAS lies in this regime, the $k_{\perp}$-factorized result will be used instead of the full CGC for computational reasons.

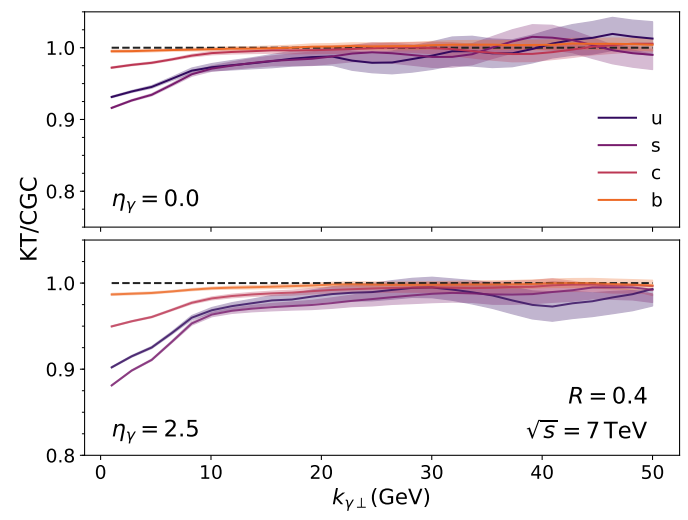

Figure 1. Ratios of the $k_{\perp}$-factorized results to the full CGC for an energy of $\sqrt{s}=7 \mathrm{TeV}$ and an isolation cut $R=0.4$. The band represents the numerical error estimate from integrating the cross section using the VEGAS Monte Carlo routine.

As it was said before, the NLO channel contribution can be greatly enhanced around small- $x$ regimes. This can be achieved by focusing on central rapidities and low momenta, where $x_{t} \sim$ $k_{\gamma \perp} e^{-y_{\gamma}} / \sqrt{s}$ for the inclusive cross-section. In Figure 2 we can observe that comparison for different energies at a fixed rapidity, $\eta_{\gamma}=1.0$, and going to more forward rapidities for a fixed energy,

1 For the NLO $g g \rightarrow q \bar{q} \gamma$ channel, one needs to insert two isolation cuts. 
$\sqrt{s}=7 \mathrm{TeV}$. We observe that at RHIC energies, $\sqrt{s}=0.2 \mathrm{TeV}$, the NLO contribution is quite small, maximally of $10 \%$ at lower momenta. However, at larger LHC energies, like $\sqrt{s}=7 \mathrm{TeV}$ and $13 \mathrm{TeV}$, the gluonic processes dominate, as the NLO contribution is more than $90 \%$ of the total cross-section. From these results we can conclude that gluons indeed dominate the proton initial states at these high energies.
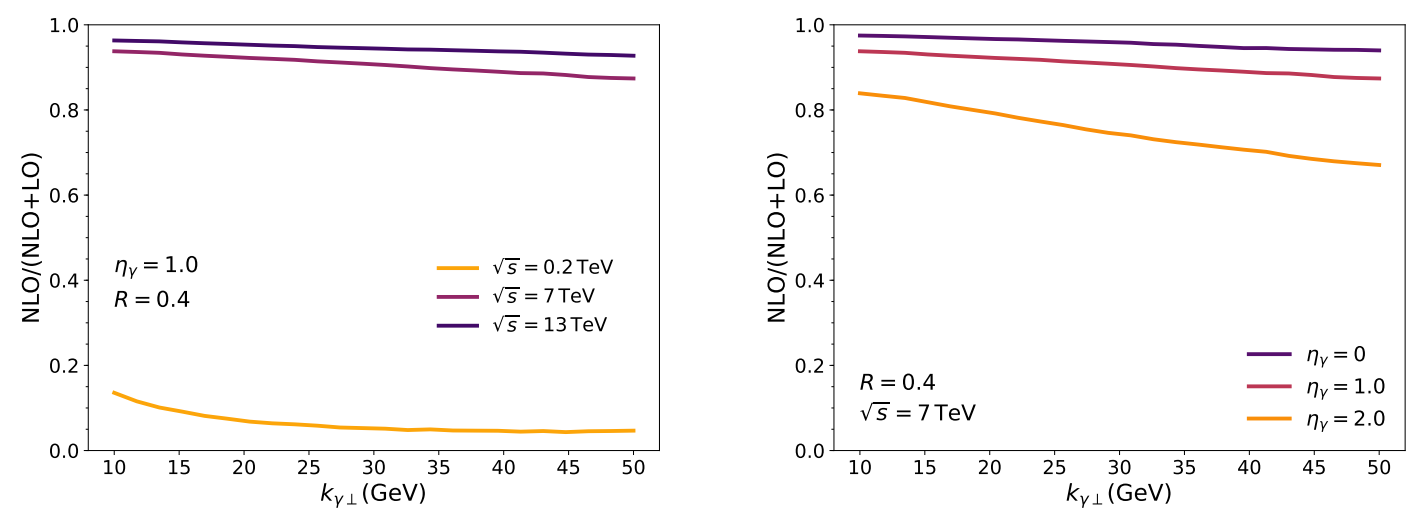

Figure 2. Fraction of the inclusive photon cross section from the NLO $g g \rightarrow q \bar{q} \gamma$ channel relative to the total NLO+LO contribution, as a function of $k_{\gamma \perp}$ for the $k_{\perp}$-factorized formula [4]. The left panel shows the collision energy dependence at $\sqrt{s}=0.2,7,13 \mathrm{TeV}$ for $\eta_{\gamma}=1.0$. The right panel shows the photon rapidity dependence at $\eta_{\gamma}=0,1.0,2.0$ for $\sqrt{s}=7 \mathrm{TeV}$. In both cases, $R=0.4$.

We will now briefly list our sources of uncertainty (for a more detailed discussion see ref. [6]), which will be included in the comparison to experimental data. First, there are $1 / N_{c}^{2}$ corrections while using the BK truncation for the dipole evolution. However, these corrections are smaller and especially suppressed for high $k_{\perp}$. Because of the inclusion of both quark and photon higher momenta, we get a competition between large- $k_{\perp}$ (DGLAP regime) and small- $x$ ( $k_{\perp}$-factorization) logarithms. The agreement between these two frameworks can be improved systematically. The other systematic source of uncertainty comes from the matching of the UGD to the PDF, and the fixing of the transverse area, which can have as much as $50 \%$ uncertainty. For now, we will absorb these two sources with an overall factor, $K$, which will be used to fit the data from the most central rapidity bin. We have quantified our numerical error due to the VEGAS integration to lie consistently in the $0-5 \%$ range. We also varied the quark masses for the ranges $m_{u, d}=0.003-0.007 \mathrm{GeV}, m_{s}=0.095-0.15 \mathrm{GeV}, m_{c}=1.3-1.5$ $\mathrm{GeV}$ and $m_{b}=4.2-4.5 \mathrm{GeV}$. It was observed that the cross section for $10 \mathrm{GeV}<k_{\gamma \perp}<50 \mathrm{GeV}$ varies by $5-10 \%$ for the light $u, d$, and $s$ quarks, while the heavier $c$ and $b$ quarks have small variations of order $0-5 \%$. For this reason, we include a very conservative overall $10 \%$ due to quark mass variation. Because of both uncertainties, we quantify our errorbands with an overall $15 \%$, for the comparison with experimental data.

Our $k_{\perp}$-factorized results describe the LHC data at 2.76 and $7 \mathrm{TeV}$ quite well (see Figure 3). As it was stated before, an overall normalization factor was used to describe the systematics. This prefactor, $K$, was used fit the data to the most central bin, and found to be $K=2.4$. A similar value was found in the fit used to describe D-meson production in the same framework [12]. This is expected since both processes involve the same $q \bar{q}$ production channel.

\section{Conclusions}

In this work we have taken a step towards constraining the photon distributions using photons for small- $x$ physics. We calculated for the first time the complete LO+NLO inclusive cross section in the CGC framework. We also quantified the relative contribution of both LO and NLO, in terms of rapidity and center-of-mass energy variables, and found that as expected, gluonic processes rapidly rise with lower $x$ values. The full cross-section, calculated in the $k_{\perp}$-factorized limit exhibits good 
agreement with the experimental data available for $p p$ collisions from CMS and ATLAS, within systematic uncertainty bands. In future studies, we will extend our results to minimum bias $p A$ data as well as $p p$ and $p A$ collisions with high particle multiplicity.

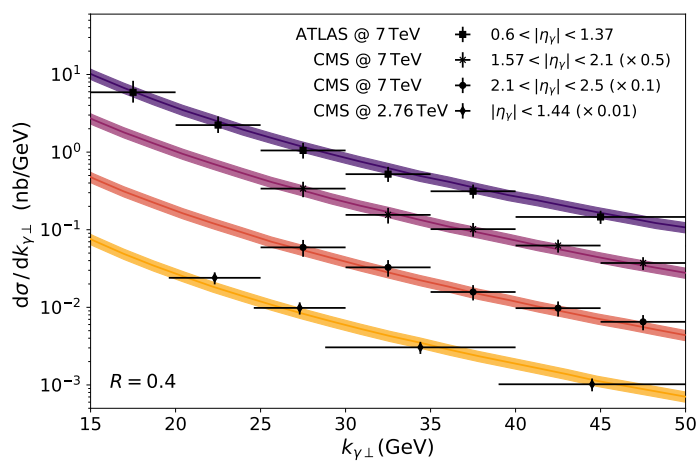

Figure 3. Comparison to the CMS and ATLAS $p+p$ photon data at $\sqrt{s}=2.76,7 \mathrm{TeV}$ [13-15]. The central line is obtained by multiplying our numerical results with a $K$-factor of $K=2.4$. The band represents a $15 \%$ a systematic uncertainty of our calculation across several rapidity bins.

Acknowledgments: We thank Kevin Dusling for providing us his rcBK code. S. B. thanks Yoshitaka Hatta for discussions and Davor Horvatić and Nenad Mijić for their help with the numerical procedures. S. B. is supported by a JSPS postdoctoral fellowship for foreign researchers under Grant No. 17F17323. S. B. was previously supported by the European Union Seventh Framework Programme (FP7 2007-2013) under grant agreement No. 291823, Marie Curie FP7-PEOPLE-2011-COFUND NEWFELPRO Grant No. 48. S. B. also acknowledges the support of the HRZZ Grant No. 8799 for computational resources. O. G. wants to thank J. Berges and A. Mazeliauskas for discussions. K. F. was supported by Japan Society for the Promotion of Science (JSPS) KAKENHI Grant No. 18H01211. R. V's work is supported by the U.S. Department of Energy, Office of Science, Office of Nuclear Physics, under Contracts No. DE-SC0012704 and within the framework of the TMD Theory Topical Collaboration. This work is part of and supported by the DFG Collaborative Research Centre "SFB 1225 (ISOQUANT)".

\section{References}

1. Gelis, F.; Iancu, E.; Jalilian-Marian, J.; Venugopalan, R. The Color Glass Condensate. Ann. Rev. Nucl. Part. Sci. 2010, 60, 463. arXiv:1002.0333.

2. Gelis, F.; Jalilian-Marian, J. Photon production in high-energy proton nucleus collisions. Phys. Rev. D 2002, $66,014021$.

3. Kopeliovich, B.; Tarasov, A.; Schafer, A. Bremsstrahlung of a quark propagating through a nucleus. Phys. Rev. C 1999, 59, 1609.

4. Benic, S.; Fukushima, K.; Garcia-Montero, O.; Venugopalan, R. Probing gluon saturation with next-to-leading order photon production at central rapidities in proton-nucleus collisions. JHEP 2017, 1701, 115. arXiv:1609.09424.

5. Benic, S.; Fukushima, K. Photon from the annihilation process with CGC in the $p A$ collision. Nucl. Phys. A 2017, 958, 1. arXiv:1602.01989.

6. Benić, S.; Fukushima, K.; Garcia-Montero, O.; Venugopalan, R. Constraining unintegrated gluon distributions from inclusive photon production in proton-proton collisions at the LHC.Phys. Lett. B 2019, 791, 11-16.

7. Frixione, S. Isolated photons in perturbative QCD. Phys. Lett. B 1998, 429, 369.

8. d'Enterria, D.; Rojo, J. Quantitative constraints on the gluon distribution function in the proton from collider isolated-photon data. Nucl. Phys. B 2012, 860, 311. arXiv:1202.1762.

9. Pumplin, J.; Stump, D.; Huston, J.; Lai, H.; Nadolsky, P.; Tung, W. New generation of parton distributions with uncertainties from global QCD analysis. JHEP 2002, 0207, 012.

10. Dusling, K.; Gelis, F.; Lappi, T.; Venugopalan, R. Long range two-particle rapidity correlations in A+A collisions from high energy QCD evolution. Nucl. Phys. A 2010, 836, 159. arXiv:0911.2720.

11. Ma, Y.; Venugopalan, R. Comprehensive Description of $\mathrm{J} / \Psi$ Production in Proton-Proton Collisions at Collider Energies. Phys. Rev. Lett. 2014, 113, 192301. arXiv:1408.4075. 
12. Ma, Y.; Tribedy, P.; Venugopalan, R.; Watanabe, K. Event engineering studies for heavy flavor production and hadronization in high multiplicity hadron-hadron and hadron-nucleus collisions. Phys. Rev. D 2018, 98, 074025. arXiv:1803.11093.

13. CMS Collaboration. Measurement of isolated photon production in $p p$ and $\mathrm{PbPb}$ collisions at $\sqrt{s_{N N}}=2.76$ TeV. Phys. Lett. B 2012, 710, 256. arXiv:1201.3093.

14. ATLAS Collaboration. Measurement of the inclusive isolated prompt photon cross section in $p p$ collisions at $\sqrt{s}=7 \mathrm{TeV}$ with the ATLAS detector. Phys. Rev. D 2011, 83, 052005. arXiv:1012.4389.

15. CMS Collaboration. Measurement of the Differential Cross Section for Isolated Prompt Photon Production in pp Collisions at 7 TeV. Phys. Rev. D 2011, 84, 052011. arXiv:1108.2044.

(C) 2019 by the authors. Licensee MDPI, Basel, Switzerland. This article is an open access article distributed under the terms and conditions of the Creative Commons Attribution (CC BY) license (http://creativecommons.org/licenses/by/4.0/). 